\title{
Yinzhihuang attenuates ANIT-induced intrahepatic cholestasis in rats through upregulation of Mrp2 and Bsep expressions
}

\author{
Qiao-Qun Ou',2, Xin-Hua Qian', Ding-You Li ${ }^{3}$, You-Xiang Zhang ${ }^{2}$, Xia-Nan Pei', Jin-Wen Chen ${ }^{1}$ and Li Yu ${ }^{2}$
}

BACKGROUND: The treatment of intrahepatic cholestasis has been limited, and development of an effective drug is needed. Clinical studies have shown that Yinzhihuang $(\mathrm{YZH})$, a traditional Chinese decoction, enhances bilirubin clearance. The goal of this study was to determine the protective effect of $\mathrm{YZH}$ on experimental intrahepatic cholestasis in young rats and to explore its underlying molecular mechanisms.

METHODS: Intrahepatic cholestasis in rats was induced by a-naphthylisothiocyanate (ANIT) on days 1 and 8. The rats received $\mathrm{YZH}$, ursodeoxycholic acid (UDCA), or vehicle for $9 \mathrm{~d}$ and were killed on either day 3 or day 10 . Serum biomarkers, liver histology, and the distribution of protein and mRNA expression of Mrp2 and Bsep were analyzed.

RESULTS: $Y Z H$ treatment resulted in decreased levels of serum biomarkers except $\gamma$-glutamyl transpeptidase, attenuated liver histological injuries, increased protein expressions of Mrp2 and Bsep, and upregulated expressions of Mrp2 and Bsep mRNAs. The effects of YZH on serum biomarkers (aminotransferase, alanine aminotransferase, and direct bilirubin), liver histology, and Mrp2 mRNA expressions were significantly greater and earlier than those of UDCA.

CONCLUSION: Our results suggest that YZH has protective effect against ANIT-induced intrahepatic cholestasis in rats, through upregulation of Mrp2 and Bsep expressions.

C holestasis is defined as a decrease in bile flow due to impaired secretion by hepatocytes or obstruction of bile flow through intra- or extrahepatic bile ducts, resulting in accumulation of bile acids and other chemicals in liver and blood (1). It affects $\sim 1$ in 2,500 live births (2). Intrahepatic cholestasis is usually caused by viruses, drugs, and metabolic or genetic disorders (3). To date, there has been no effective medical treatment for intrahepatic cholestasis. Even though ursodeoxycholic acid (UDCA) is the mainstay for the treatment of various cholestatic disorders (4), most patients only exhibit partial response and eventually progress to cirrhosis and liver failure (5-7). Therefore, the development of an effective drug for intrahepatic cholestasis is desperately needed.
Yinzhihuang (YZH), a traditional Chinese decoction, contains extracts from Yin Chin (Artemisia annual L) and three other herbs (Gardenia jasminoides Ellis, Rheum Palatum L, and Scutellaria baicalensis) (8-10). It has been widely used to prevent and treat neonatal jaundice for centuries in Asia. Clinical studies have shown that YZH oral liquid is safe in premature infants and enhances bilirubin clearance in newborns and infants (11-13). In addition, YZH has been shown to be somewhat more effective than phenobarbital on bilirubin clearance (10). Recent progress has shown that cholestasis is associated with complex transcriptional and posttranscriptional alterations of hepatobiliary transporters and enzymes participating in bile formation $(3,14)$. It has been demonstrated that YZH is a potent activator of constitutive androstane receptor, a key regulator of the bilirubin clearance (9). However, it is not known whether YZH regulates the hepatobiliary transporters, multidrug resistant associated protein 2 (Mrp2), and bile salt export pump (Bsep).

In rodents, $\alpha$-naphthylisothiocyanate (ANIT) is widely used to induce an animal model of intrahepatic cholestasis. ANITinduced cholestasis is characterized by elevation of serum transaminases and bile acids, necrosis of hepatobiliary cells, and infiltration of inflammatory cells, followed by obstruction and proliferation of bile ducts (15-18). The biomarkers for liver injury and cholestasis peak at $48 \mathrm{~h}$ after ANIT treatment and recover a week later. ANIT administered weekly can prolong the experimental time and imitate chronic cholestasis $(19,20)$.

The purpose of this study was to assess the effect of YZH on ANIT-induced intrahepatic cholestasis in rats and to further investigate its underlying molecular mechanism involving Mrp2 and Bsep expressions.

\section{RESULTS}

Macroscopic Changes of Rats

Rats were randomly assigned to receive ANIT or the same volume of sesame oil (normal control group), weekly on days 1 and 8. The ANIT-treated rats were randomly gavaged with YZH (ANIT+YZH group), UDCA (ANIT+UDCA group), or saline (ANIT+saline group) on days 1 through 10 . The rats

'Department of Neonatology, Nanfang Hospital, Southern Medical University, Guangzhou, China; ${ }^{2}$ Department of Pediatrics, Guangzhou First People's Hospital, Guangzhou Medical University, Guangzhou, China; ${ }^{3}$ Division of Pediatric Gastroenterology, Department of Pediatrics, Children's Mercy Hospital, University of Missouri Kansas City School of Medicine, Kansas City, Missouri. Correspondence: Xin-Hua Qian (qxh_nfyy@163.com)

Received 13 April 2015; accepted 16 September 2015; advance online publication 6 January 2016. doi:10.1038/pr.2015.252 


\section{Articles | ouetal.}

that received ANIT treatment displayed icteric ears and yellowish urine, reduced activity, poor appetite, and slower or even negative weight gain. Those rats in both ANIT+YZH and ANIT+UDCA groups displayed less icteric ears and urine and better activity and appetite.

\section{Serum Hepatic Biomarkers in Rats Receiving Different Treatments}

To study the liver biomarker changes after YZH and UDCA treatment, serum aspartate aminotransferase (AST), alanine aminotransferase (ALT), $\gamma$-glutamyl transpeptidase $(\gamma \mathrm{GT})$, alkaline phosphatase (ALP), direct bilirubin (DB), and total bile acid (TBA) were analyzed, and all of the six biomarkers were significantly increased in ANIT+saline group on day 3 and remained at high levels on day 10, compared to those in normal control group (Figure 1).

Compared to the ANIT+saline group, YZH treatment significantly attenuated AST and ALT elevations on the 3rd day (D3) and 10th day (D10), whereas UDCA treatment only significantly attenuated AST and ALT increases at the later time point on D10; serum $\gamma$ GT was significantly decreased only on D10 but not on D3 and serum DB levels were significantly reduced on both D3 and D10 in both YZH- and UDCA-treated groups; serum ALP and TBA were decreased in the YZH treatment group on D3 and D10, but only on D10 in the UDCA treatment group (Figure 1). In addition, reduction of AST and ALT by YZH treatment was significantly greater than that by UDCA treatment $(P<0.05)$.
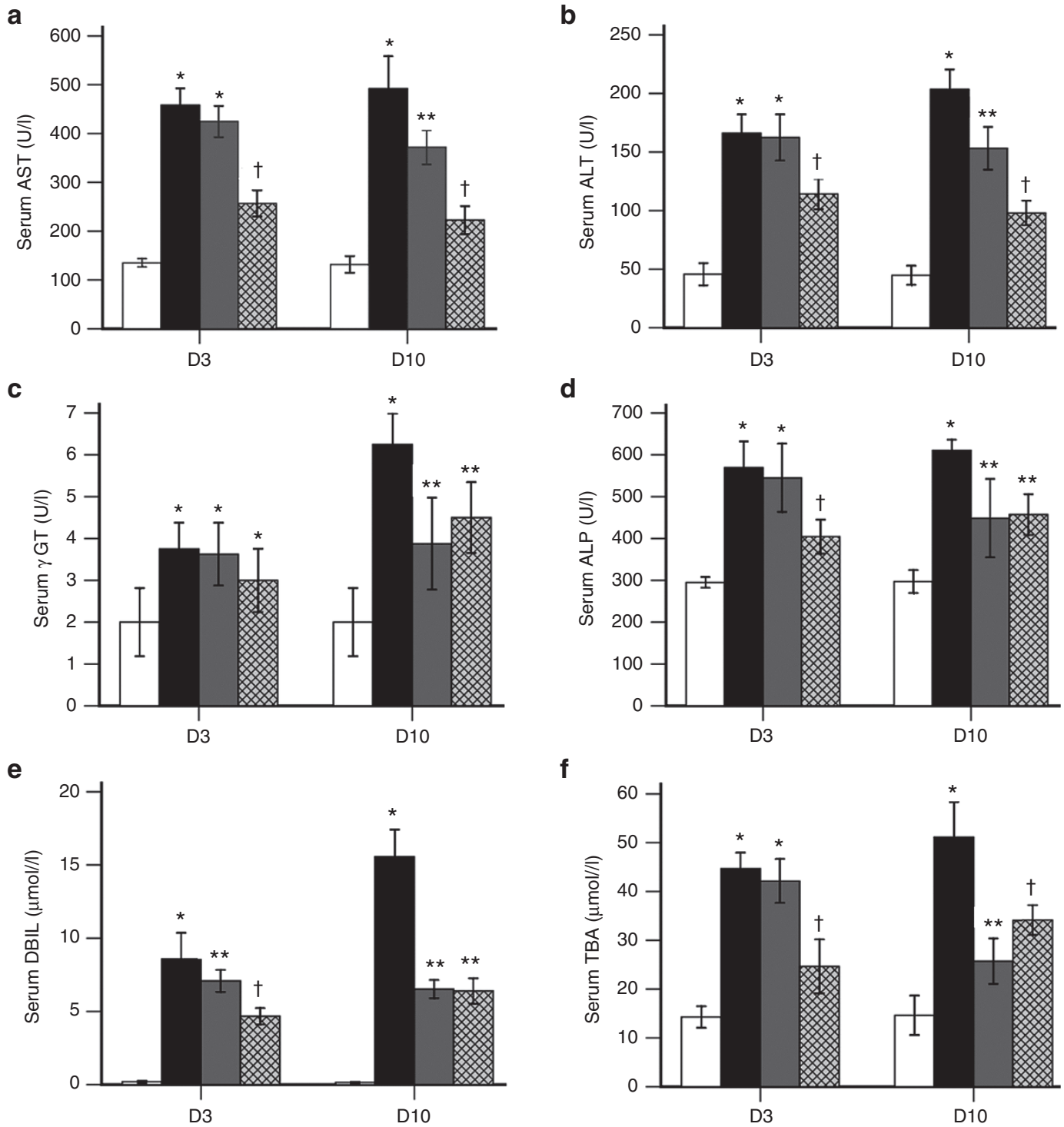

Figure 1. Effects of Yinzhihuang (YZH) and ursodeoxycholic acid (UDCA) on serum hepatic biomarkers in $\alpha$-naphthylisothiocyanate (ANIT)-induced cholestasis rats. Rats were randomly assigned to normal control (white bars), ANIT+saline (black bars), ANIT+UDCA (gray bars), or ANIT+YZH (hatched bars) groups. Blood samples were collected $48 \mathrm{~h}$ after the ANIT treatment on 3rd (D3) and 10th (D10) days and assayed for (a) AST, (b) ALT, (c) $\gamma \mathrm{GT}$, (d) ALP, (e) DB, and (f) TBA. Data were expressed as mean \pm SEM. ${ }^{*} P<0.05$ compared with normal control group; ${ }^{* *} P<0.05$ compared with ANIT+saline group; ${ }^{+} P<0.05$ compared with both ANIT+UDCA group and ANIT+saline group. ALT, alanine aminotransferase; ALP, alkaline phosphatase ; AST, aspartate aminotransferase; DB, direct bilirubin; $\gamma \mathrm{GT}$, $\gamma$-glutamyl transpeptidase; TBA, total bile acid. 


\section{Liver Histological Changes in Rats}

Liver sections were examined for hepatic necrosis, inflammation, enlarged portal fields, and proliferation (Figure 2). Compared with the normal control group, the ANIT+saline group showed hepatocyte necrosis and degenerative change with inflammatory cell infiltration, enlarged portal fields, ductule proliferation, and fibroblast proliferation. These changes were more visible at D10 (Figure 2, hematoxylin and eosin staining) than those at D3. The ANIT+YZH group and the ANIT+UDCA group had less necrotic and degenerative changes as well as less inflammatory cell infiltration. Masson's trichrome staining showed that collagen depositions were increased in the ANIT+saline group in comparison to normal controls. YZH, not UDCA, treatment dramatically decreased collagen depositions (Figure 2, Masson's trichrome staining).

Liver injury was scored according to previous literature $(21,22)$, and the moderate-to-severe injury affecting more than $25 \%$ is considered as significant liver injury. Our study also showed that 87.5 and $100 \%$ of rats in the ANIT group had significant liver injury on D3 and D10, respectively. In UDCAand $\mathrm{YZH}$-treated groups, $37.5 \%$ of rats showed significant liver injury on D3. On D10, the rates were 37.5 and $25 \%$, respectively, in the two groups. However, there were no significant difference between UDCA- and YZH-treated groups.

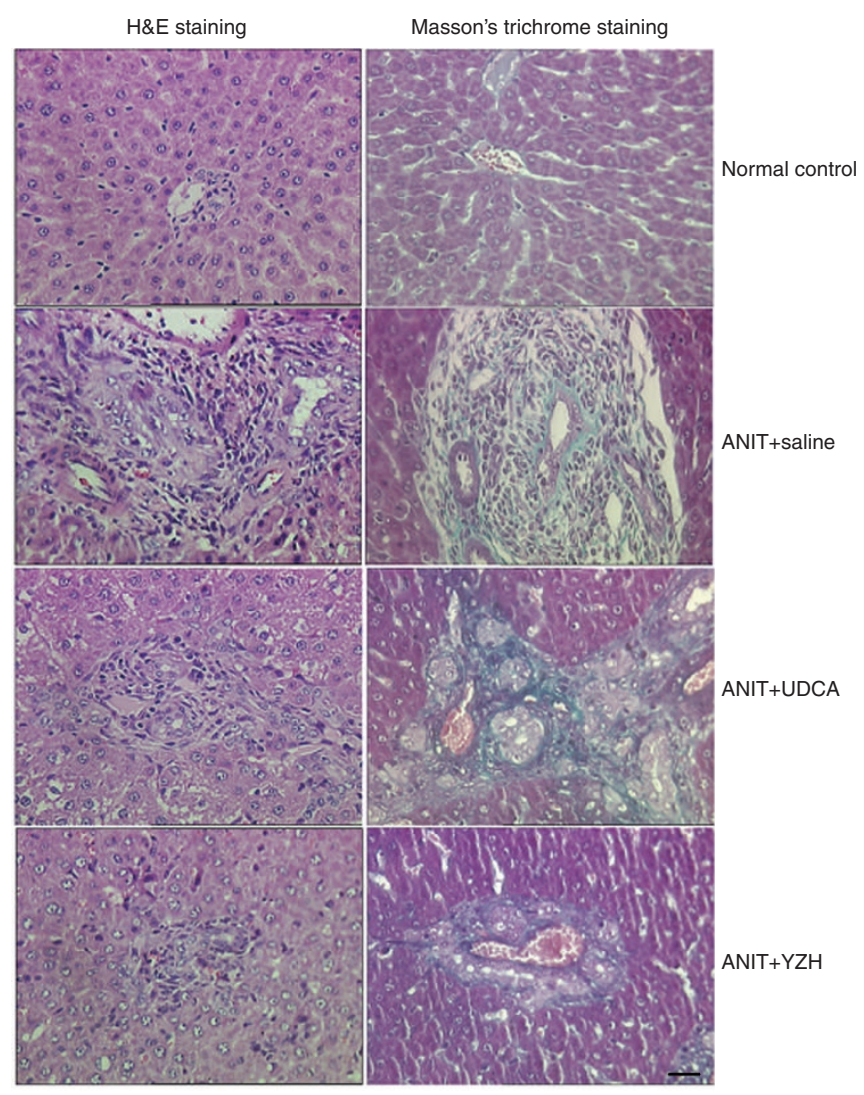

Figure 2. Effects of Yinzhihuang (YZH) and ursodeoxycholic acid (UDCA) on $\alpha$-naphthylisothiocyanate (ANIT)-induced liver injury. Rats were randomly assigned to normal control, ANIT+saline, ANIT+YZH, or ANIT+UDCA groups. Liver tissues were obtained on the 10th day, prepared, stained with H\&E or Masson's trichrome, and examined by light microscopy. Magnification: $\times 200 ; \mathrm{bar}=50 \mu \mathrm{m}$. H\&E, hematoxylin and eosin.

\section{Mrp2 and Bsep Protein Distribution in Liver Tissues of Rats}

Immunohistochemical techniques were used to detect the distribution and protein expression of hepatobiliary transporters, Mrp2 and Bsep. Normally, Mrp2 and Bsep protein are localized in the basolateral membrane of hepatic cells as a distinct ribbon-like zone around the cell. The integrated optical density was taken with the Image-Pro Plus 6.0 software to qualify the stained density. Protein expressions of Mrp2 and Bsep in the ANIT+saline group were significantly decreased in comparison to normal controls. In UDCA- and YZH-treated groups, protein expressions of Mrp2 and Bsep were increased significantly when compared with the ANIT+saline group (Figures 3 and 4).

\section{Mrp2 and Bsep mRNA Expressions in Liver Tissues of Rats}

Quantitative real-time fluorescence PCR showed that mRNA expressions of Mrp2 and Bsep corresponded with the results of protein expressions (Figure 5). ANIT treatment resulted in a significant reduction in liver Mrp2 and Bsep mRNA expressions. On D3, the Mrp2 mRNA expression in UDCA and YZH groups was upregulated to 1.7-fold and 2.1-fold, respectively, as compared to the ANIT+saline group. On D10, Mrp2 mRNA expression in UDCA and YZH groups was increased to 2.2fold and 2.6-fold, respectively, as compared to the ANIT+saline group. The effect of YZH on Mrp2 mRNA expression at both time points was significantly higher than that of UDCA. Similarly, both UDCA and YZH treatments increased Bsep mRNA expression to 1.5 -fold on D3 and 2.1-fold on D10, respectively, compared with that in the ANIT+saline group.

\section{DISCUSSION}

Cholestasis is a pathological condition caused by impairment of hepatic bile flow. Toxic bile acid accumulation and inflammation leads to liver injury, fibrosis, and hepatocirrhosis. Treatment options for cholestatic liver injury are very limited $(4,23)$. UDCA, the mainstay of therapy, has produced conflicting data (5-7). Hence, the development of a new effective drug is needed. This study demonstrated a beneficial effect of $\mathrm{YZH}$ on ANIT-induced cholestasis in young rats. YZH treatment attenuated serum levels of hepatic biomarkers and liver histological changes, prevented fibrosis, and upregulated expressions of Mrp2 and Bsep.

ANIT-induced cholestasis is characterized by elevation of serum transaminases and bile acids, necrosis of hepatobiliary cells, and infiltration of inflammatory cells, followed by obstruction and proliferation of bile ducts $(17,18)$. ANIT selectively damages bile-duct epithelial cells and causes cholangitis and subsequent intrahepatic cholestasis (24). The biomarkers for liver injury and cholestasis peak at $48 \mathrm{~h}$ after ANIT treatment and recover a week later. ANIT administered weekly can prolong the experimental time and imitate chronic cholestasis $(19,20)$. So ANIT given weekly and rats killed at $48 \mathrm{~h}$ after ANIT treatment completion were chosen in this study. Our results showed that the biomarkers for liver injury and cholestasis were significantly enhanced at $48 \mathrm{~h}$ after ANIT treatment, in agreement with a previous report (16). 


\section{Articles | Ouetal.}
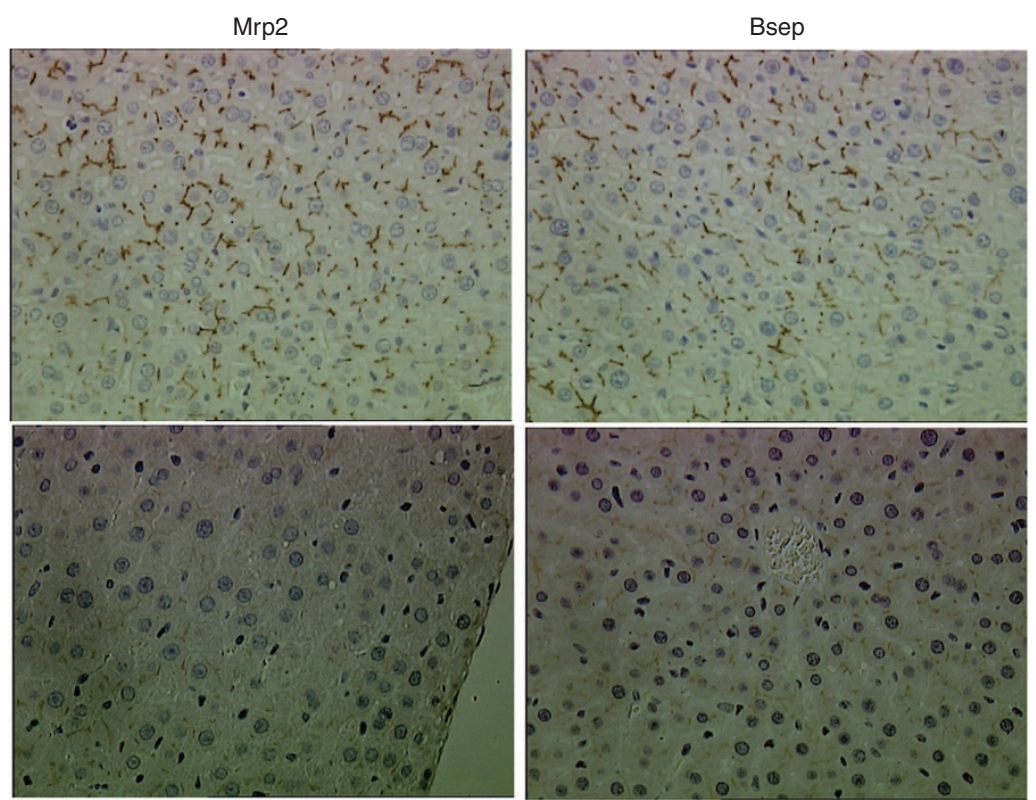

Normal control
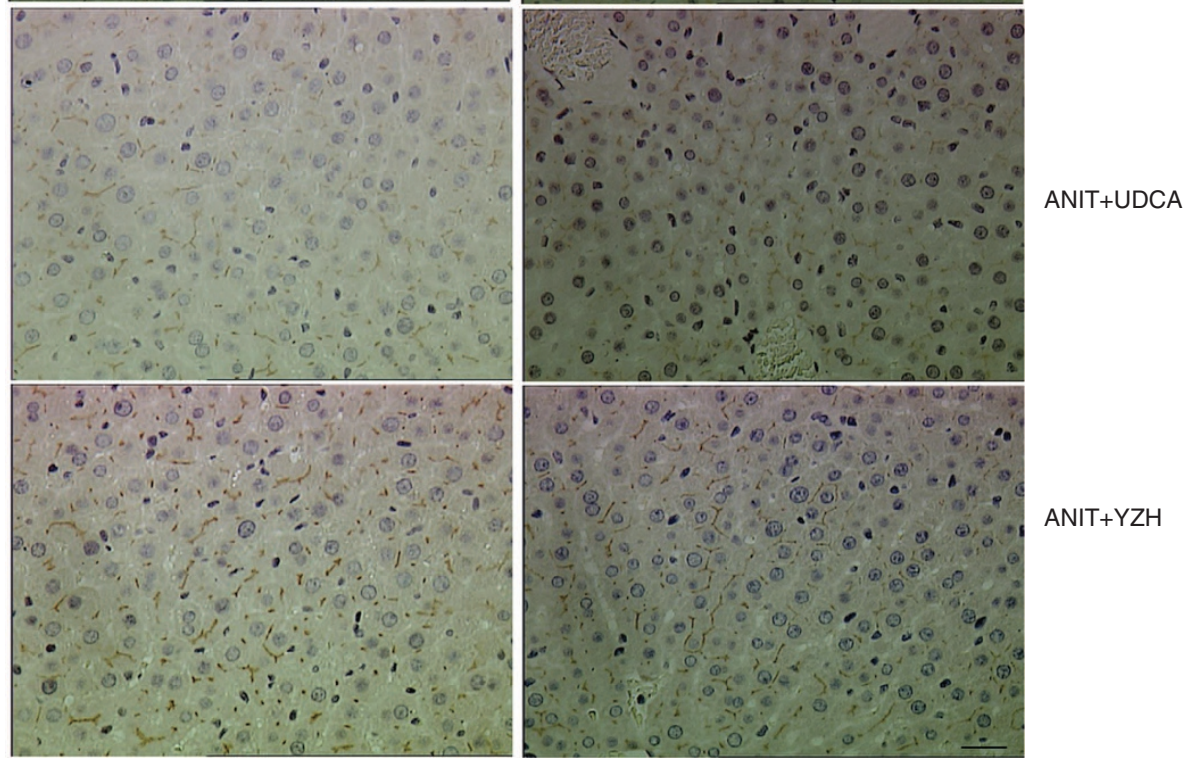

ANIT+saline

ANIT+UDCA

ANIT+YZH

Figure 3. Protein expressions of hepatobiliary transporters, Mrp2 and Bsep, in liver tissues of rats. Rats were randomly assigned to normal control, ANIT+saline, ANIT+YZH, or ANIT+UDCA groups. Liver tissues were obtained on the 10th day, prepared, stained immunohistochemically for Mrp2 and Bsep. Magnification: $\times 200 ;$ bar $=50 \mu \mathrm{m}$.

The hepatic histology showed necrosis and degeneration with severe inflammatory cell infiltration and biliary hyperplasia. Moreover, the liver fibrosis with cholestasis was observed at D10 after prolonged ANIT treatment. These data confirmed that the intrahepatic cholestasis was induced successfully with ANIT in our animal model.

In this study, we demonstrate the protective effect of $\mathrm{YZH}$ against ANIT-induced liver damage with intrahepatic cholestasis. First, we assessed the effects of YZH on serum biomarkers of liver damage and cholestasis. YZH treatment significantly attenuated ANIT-induced increases in serum concentrations of ALT, AST, ALP, $\gamma \mathrm{GT}, \mathrm{DB}$, and TBA, indicating its protective effects on hepatobiliary cells. Furthermore, we found that the effect of YZH occurred earlier and was stronger than that of UDCA. Second, we evaluated the effects of YZH on histological changes of liver damage and cholestasis. YZH treatment dramatically attenuated the necrotic and degenerative changes of hepatobiliary cells, the bile duct proliferation, and the inflammatory cell infiltration induced by ANIT. Most importantly, YZH treatment was clearly associated with less histological changes than UDCA treatment. These changes were consistent with the results of serum biomarkers. Another interesting result from Masson's staining indicated that the inhibitory effect of YZH on fibrosis was more apparent than that of UDCA. This antifibrosis effect of YZH is crucial since fibrosis is indicative of deterioration of liver function and hepatocirrhosis. Our study suggests that $\mathrm{YZH}$ protects against ANIT-induced intrahepatic cholestasis by decreasing hepatobiliary cell damage, attenuating inflammatory cell infiltration, and promoting clearance of accumulated bilirubin and bile acid. 
a

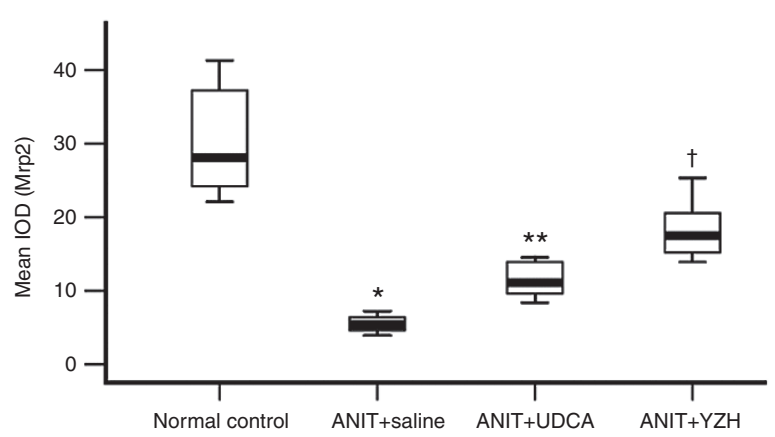

b

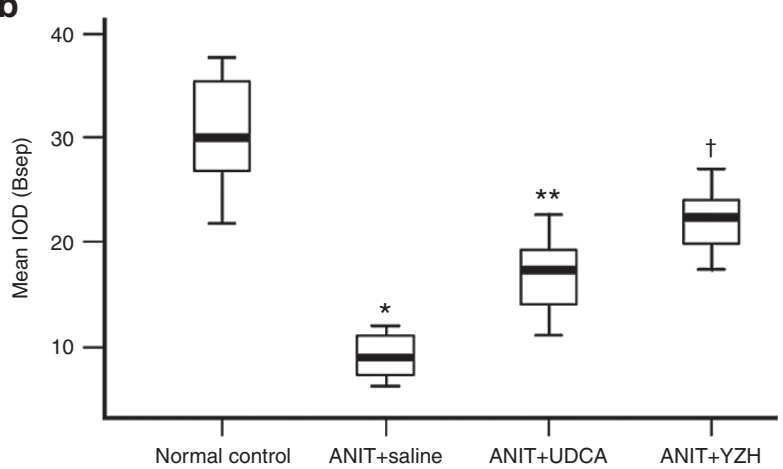

Figure 4. Quantitative analyses of protein expressions of hepatobiliary transporters, Mrp2 and Bsep, in liver tissues of rats. Rats were randomly assigned to normal control, ANIT+saline, ANIT+YZH, or ANIT+UDCA groups. Liver tissues were obtained on the 10th day, prepared, stained immunohistochemically for (a) Mrp2 and (b) Bsep. The integrated optical density (IOD) as density of positive staining was measured by Image-Pro Plus 6.0 software. The mean IOD represents the expression level of Mrp2 or Bsep. ${ }^{*} P<0.05$ compared with normal control group; ${ }^{* *} P<0.05$ compared with ANIT+saline group; ${ }^{\dagger} P<0.05$ compared with both ANIT+UDCA groups and ANIT+saline groups.

a

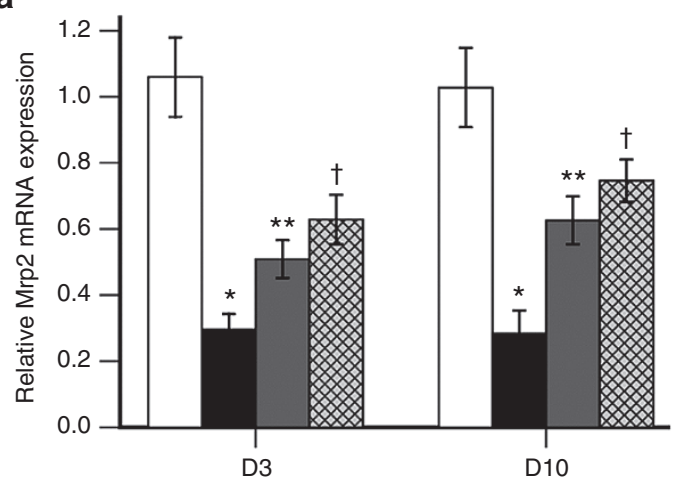

b

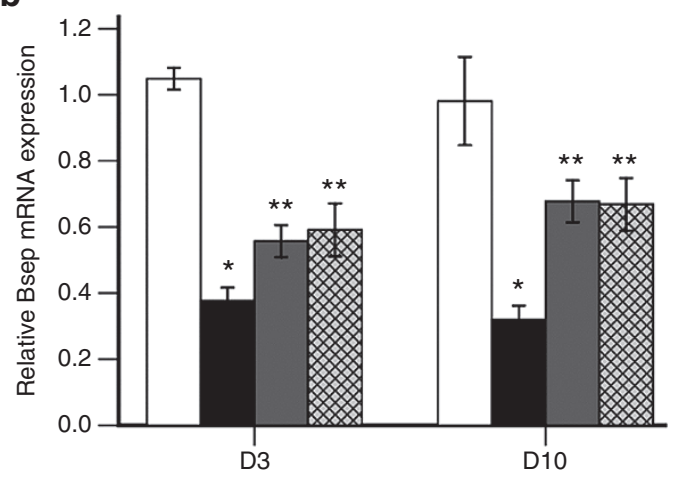

Figure 5. Relative mRNA expressions of hepatobiliary transporters, Mrp2 and Bsep, in liver tissues of rats. Rats were randomly assigned to normal control (white bars), ANIT+saline (black bars), ANIT+UDCA (gray bars) or ANIT+YZH (hatched bars) groups. Liver tissues were obtained on the 3rd (D3) and 10th (D10) days and (a) Mrp2 and (b) Bsep mRNA expressions were determined by qRT-PCR. Data were expressed as mean \pm SEM. * $P<0.05$ compared with normal control group; ${ }^{* *} P<0.05$ compared with ANIT+saline group; ${ }^{\dagger} P<0.05$ compared with both ANIT+UDCA group and ANIT+saline group. qRT-PCR, quantitative real-time fluorescence PCR.

$\mathrm{YZH}$ is extracted from four different plants: A. annual $\mathrm{L}$, G. jasminoides Ellis, R. Palatum L, and S. baicalensis. It contains many components that may have different properties and affect various metabolic enzymes, transporters, and receptors. The herb-drug interactions are so complicated that it is difficult to understand its mechanism of action. However, in Chinese and other traditional medicines, disease is thought to be a consequence of imbalances in the body, and it is necessary to incorporate multiple components into a therapeutic approach to restore the balance $(24,25)$. YZH is a typical example of such a combination. Moreover, modern biochemical analyses have confirmed that the combination of these herbs achieved an ideally synergistic and maximum therapeutic outcome as compared to any component used individually (26). Previous studies reported that $A$. annual $\mathrm{L}$ and $G$. jasminoides Ellis exhibited antioxidant and anti-inflammatory properties (26-29); R. Palatum L was proven to ameliorate liver fibrosis (30-32); and S. baicalensis was identified not only to have antioxidant and anti-inflammatory properties but also antifibrosis effects and modulation of the immune response $(33,34)$. The synergistic effect of the various components may explain why YZH exerts somewhat more potent protective effects against ANIT-induced cholestasis than UDCA.

Recent studies have demonstrated that hereditary mutations of transporter genes or exposure to cholestatic injury (e.g., drugs, hormones, proinflammatory cytokines, and biliary obstruction) result in reduced expression and function of hepatobiliary transport systems, which play an important role in pathogenesis of cholestasis $(3,14)$. Hepatobiliary transporters, Mrp2 and Bsep, are members of ATP-binding cassette-dependent transporters. Mrp2 exports glutathione and numerous other organic anions into bile, and Bsep is relatively specific for the biliary transport of bile acids. Impaired expressions and/or functions of both of these transporters cause significant cholestasis $(24,35,36)$. On the other hand, Mrp2 and Bsep are downregulated in cholestasis in rats as well as humans (37), leading to cytotoxic bile acid accumulation and liver damage. In this study, we observed reductions of Mrp2 and Bsep protein expression and downregulations of Mrp2 and Bsep mRNA in livers of rats after ANIT treatment. YZH treatment partially 
but significantly reversed ANIT-induced decreases of Mrp2 and Bsep protein and mRNA expressions. Moreover, we found that the effect of YZH on Mrp2 protein and mRNA expression was more potent than that of UDCA, whereas YZH and UDCA had similar effects on Bsep protein and mRNA expressions. It has been shown that Mrp2 expression is a prerequisite for this cholestasis in rats since ANIT-glutathione conjugate is transported by Mrp2 into bile and concentrated in the canaliculus, and the dissociated ANIT selectively damages bile-duct epithelial cells, resulting in cholangitis and subsequent intrahepatic cholestasis $(24,38)$. We found that Mrp2 is preserved in the YZH-treatment group, a fact that can also contribute to prolonged toxic effects via the intrahepatic cycling over the hepatocytic membrane. Our results suggest that a molecular mechanism of YZH may involve stimulation of Mrp2 (increasing bile acid-independent bile flow) and Bsep (increasing bile acid-dependent bile flow), which help to reduce the accumulation of toxic biliary constituents in cholestasis.

In conclusion, we have demonstrated that the oral administration of YZH protected against ANIT-induced intrahepatic cholestasis in rats and the underlying molecular mechanism involved upregulation of hepatobiliary transporters, Mrp2 and Bsep. The protective effect of YZH was more potent than that of UDCA. These results suggest that YZH may provide an alternative treatment for intrahepatic cholestasis. However, further study is needed to clarify which specific component of YZH is most effective and to identify whether YZH affects other bile acid transporters and nuclear receptors involving in metabolic regulatory function of the bile acids.

\section{METHODS}

\section{Chemicals and Drugs}

ANIT was purchased from Sigma Aldrich (St Louis, MO). YZH oral liquid was provided by China Resources Sanjiu Medical \& Pharmaceutical (Beijing, China). UDCA was obtained from Dr Falk Pharma (Frei Burg, Germany). The hepatic biomarker kits were obtained from Beckmancoulter Lab System (Suzhou, China). Mrp2 and Bsep antibody were obtained from Santa Cruz Biotechnologies (Santa Cruz, CA). The qRT-PCR kit was obtained from TaKaRa (Dalian, China). The primer of Mrp2 and Bsep were from Sangon Biotech (Shanghai, China). The other chemicals were obtained from commercial sources and of reagent grade.

\section{ANIT-Induced Intrahepatic Cholestasis in Rats}

All animal procedures were performed according to the guidelines for humane treatment of animals set by the Association of Laboratory Animal Science and were approved by Southern Medical University Animal Care and Use Committee.

All studies were performed with male SD rats aged $4-5 \mathrm{wk}$, weighting $150-180 \mathrm{~g}$. Rats were housed at an ambient temperature of $22 \pm 2$ ${ }^{\circ} \mathrm{C}$ with alternating 12 -h light/dark cycles and were provided water and food ad libitum. They were randomly assigned to receive ANIT orally at a dose of $80 \mathrm{mg} / \mathrm{kg}(0.5 \mathrm{ml} / \mathrm{kg}$ in sesame oil, ANIT group) or the same volume of sesame oil (normal control group), on days 1 and 8. Clinical symptoms of rats were observed every day, and body weight was recorded twice per week.

\section{Treatment of Intrahepatic Cholestasis in Rats With YZH and UDCA}

The ANIT-treated rats were randomly divided into three groups: ANIT + YZH group $(n=16)$, receiving YZH oral liquid $15 \mathrm{ml} / \mathrm{kg}$ by gavage once daily; ANIT+UDCA group $(n=16)$, receiving UDCA $100 \mathrm{mg} / \mathrm{kg}$ by gavage once daily; and ANIT+saline group $(n=16)$, receiving only normal saline $15 \mathrm{ml} / \mathrm{kg}$ once daily. The rats in the normal control group $(n=8)$ were also given normal saline $15 \mathrm{ml} / \mathrm{kg}$ once daily. Daily treatment with YZH, UDCA, or saline started on day 1 and continued until day 10. Half of the rats in each group were sacrificed under chloral hydrate anesthesia on D3 and the remaining ones on D10 of treatment. Blood samples and liver tissues were collected $48 \mathrm{~h}$ after ANIT treatment (on D3 and D10) for further analysis.

\section{Serum Hepatic Biomarker Assays}

Serum hepatic biomarkers, including AST, ALT, $\gamma$ GT, ALP, DB, and TBA, were analyzed by standard enzymatic-colorimetric assays using commercial assay kits in accordance with the manufacturer's protocol.

\section{Histopathological Examinations}

Liver tissues were fixed in $10 \%$ neutral buffered formalin prior to routine processing and paraffin embedding. The liver tissues were sliced into $5-\mu \mathrm{m}$ sections and stained with hematoxylin and eosin or Masson's trichrome. The stained sections were examined by a trained pathologist under light microscope for the presence and severity of hepatocellular degeneration and necrosis. Liver injury was scored according to previous literature as follows: grade 0 , no injury; grade 1 , minimal injury involving single to few hepatocytes; grade 2 , mild injury affecting $10-25 \%$ of hepatocytes; grade 3 , moderate injury affecting $25-40 \%$ of hepatocytes; grade 4 , marked injury affecting more than $40 \%$ of hepatocytes. Scores higher than grade 2 are considered as significant liver injuries $(21,22)$.

\section{Immunohistochemical Analysis of Mrp2 and Bsep Protein Expression}

Immunohistochemical stains for Mrp2 and Bsep were performed on the paraffin-embedded liver sections according to the manufacturer's protocol. Briefly, liver sections were de-paraffinized and rehydrated. Nonenzymatic antigen retrieval was carried out in $0.1 \mathrm{~mol} / \mathrm{l}$ boiling citrate buffer ( $\mathrm{pH}$ 6.0) for $3 \mathrm{~min}$ in a pressure cooker. Mrp2 mouse monoclonal antibody and Bsep mouse monoclonal antibody were incubated at a dilution of $1: 100$ for $1 \mathrm{~h}$ at $37^{\circ} \mathrm{C}$, linking with biotinylated streptavidin-horseradish peroxidase rabbit antibody against mouse IgG for $20 \mathrm{~min}$, developing chromogen with diaminobenzidine, and counterstaining with hematoxylin. The immunostained slides were evaluated by light microscopy. The integrated optical density as density of positive staining was measured by Image-Pro Plus 6.0 software (Media Cybernetics, Bethesda, MD) as described previously (39). The mean integrated optical density represents the expression level of Mrp2 or Bsep.

\section{RNA Isolation and Quantitative Real-Time Fluorescence PCR}

Frozen liver samples were homogenized in ISOGEN reagent. Total RNA was isolated using RNA isolation kits according to the manufacturer's protocol and quantified by using ultraviolet absorbance at $260 \mathrm{~nm}$. Integrity of samples was confirmed by gel electrophoresis. Gene-specific primer sequences for Mrp2, Bsep, and $\beta$-actin were as follows: Mrp2 (228bp), 5'-GCCACACTACACGGACTG AA-3' $5^{\prime}$-ACCGATCAGC AACTTCAGCA-3'; Bsep (225bp) 5'-TCTCGTGGAGAGAACAA CGC A-3', 5'-CACCACTCCTTGTGACACGACA-3' (40); $\beta$-actin (154bp), 5'-CACCCGCGAGTACAACCTTC-3', 5'-CCCATACCCA CCATCACACC- $3^{\prime}$. The cDNA was synthesized by the reverse transcription reaction as follows: $1 \mu \mathrm{g}$ of total RNA was added, it was incubated at $65^{\circ} \mathrm{C}$ for $5 \mathrm{~min}$, and then, the reaction mixture has been kept on the ice for $3 \mathrm{~min}$. A standard curve was prepared using RNA from the intact rat liver transcribed in the same way. After the first-strand cDNA synthesis, PCR reactions were performed. Thermocycling for each reaction was performed using the PCR kit. After $10 \mathrm{~min}$ of initial denaturation at $95{ }^{\circ} \mathrm{C}$, the cycling conditions of 40 cycles consisted of denaturation at $95{ }^{\circ} \mathrm{C}$ for $15 \mathrm{~s}$, annealing at $60{ }^{\circ} \mathrm{C}$ for $30 \mathrm{~s}$ and extension at $72{ }^{\circ} \mathrm{C}$ for $15 \mathrm{~s}$. Each gene was repeated three times. Fluorescence measurement of samples was done at the end of each extension step. Analyzing software produced the standard curve by measuring the crossing point of each standard and plotting it against the logarithmic values of concentrations. After the PCR procedure, the melting temperature of each PCR product was measured to check for the unique product amplification. 


\section{Statistical Analysis}

All data were analyzed using SPSS software version 13.0 (SPSS, Chicago, IL) for Windows. Results were expressed as mean \pm SEM. Data were analyzed by ANOVA, and individual means were compared using least significant difference test. A value of $P<0.05$ was considered to be statistically significant.

\section{ACKNOWLEDGMENTS}

The authors thank Craig Friesen and Beth Lyman from Children's Mercy Hospital for their critical reading of the manuscript. The authors are also grateful to Chun-Quan Ou from Southern Medical University for the assistance in statistical analysis. The authors also thank China Resources Sanjiu Medical \& Pharmaceutical Co. Ltd., for kindly providing the Yinzhihuang oral liquid.

\section{STATEMENT OF FINANCIAL SUPPORT}

No financial assistance was received in support of this study.

Disclosure: the authors have no financial ties to products in the study and no potential or perceived conflicts of interest to report.

\section{REFERENCES}

1. Trauner M, Meier PJ, Boyer JL. Molecular pathogenesis of cholestasis. N Engl J Med 1998;339:1217-27.

2. Moyer V, Freese DK, Whitington PF, et al.; North American Society for Pediatric Gastroenterology, Hepatology and Nutrition. Guideline for the evaluation of cholestatic jaundice in infants: recommendations of the North American Society for Pediatric Gastroenterology, Hepatology and Nutrition. J Pediatr Gastroenterol Nutr 2004;39:115-28.

3. Zollner G, Trauner M. Mechanisms of cholestasis. Clin Liver Dis 2008; 12:1-26, vii.

4. Yang H, Ramani K, Xia M, et al. Dysregulation of glutathione synthesis during cholestasis in mice: molecular mechanisms and therapeutic implications. Hepatology 2009;49:1982-91.

5. Goulis J, Leandro G, Burroughs AK. Randomised controlled trials of ursodeoxycholic-acid therapy for primary biliary cirrhosis: a meta-analysis. Lancet 1999;354:1053-60.

6. Kotb MA. Review of historical cohort: ursodeoxycholic acid in extrahepatic biliary atresia. J Pediatr Surg 2008;43:1321-7.

7. Kotb MA. Ursodeoxycholic acid in neonatal hepatitis and infantile paucity of intrahepatic bile ducts: review of a historical cohort. Dig Dis Sci 2009;54:2231-41.

8. Elferink RO. Yin Zhi Huang and other plant-derived preparations: where herbal and molecular medicine meet. J Hepatol 2004;41:691-3.

9. Huang W, Zhang J, Moore DD. A traditional herbal medicine enhances bilirubin clearance by activating the nuclear receptor CAR. J Clin Invest 2004;113:137-43.

10. Yin J, Miller M, Wennberg RP. Induction of hepatic bilirubin-metabolizing enzymes by the traditional Chinese medicine yin zhi huang. Dev Pharmacol Ther 1991;16:176-84.

11. Clinical Research Collaborative Group of Yinzhihuang Oral Salution. A multicenter randomized controlled study on the efficacy and safety of Yinzhihuang oral solution for the treatment of neonatal indirect hyperbilirubinemia in term newborn infants. Chin J Pediatr 2011; 49: 663-8.

12. Yin J, Wennberg RP, Xia YC, Liu JW, Zhou HZ. Effect of a traditional Chinese medicine, yin zhi huang, on bilirubin clearance and conjugation. Dev Pharmacol Ther 1991;16:59-64.

13. Chen SP, Tian LL, Liu FL. Clinical observation of Yinzhihuang oral liquid on prevention of the premature infantile jaundice. Chin J Integr Med 2009;15:299-302.

14. Wagner M, Zollner G, Trauner M. New molecular insights into the mechanisms of cholestasis. J Hepatol 2009;51:565-80.

15. Desmet VJ, Krstulović B, Van Damme B. Histochemical study of rat liver in alpha-naphthyl isothiocyanate (ANIT) induced cholestasis. Am J Pathol 1968;52:401-21.

16. Hertzog PJ, Bhathal PS, Dorling PR, Le Page RN. Alpha-naphthyl-isothiocyanate-induced cholestasis in the rat: studies of liver plasma membrane enzymes. Pathology 1975;7:13-23.

17. Amin K, Ip C, Sato B, et al. Characterization of ANIT-induced toxicity using precision-cut rat and dog liver slices cultured in a dynamic organ roller system. Toxicol Pathol 2006;34:776-84.
18. Faiola B, Peterson RA, Kimbrough CL, Jordan HL, Cullen JM. Acute ANIT toxicity in male IL-10 knockout and wild-type mice. Toxicol Pathol 2010;38:745-55.

19. Ferreira FM, Oliveira PJ, Rolo AP, et al. Cholestasis induced by chronic treatment with alpha-naphthyl-isothiocyanate (ANIT) affects rat renal mitochondrial bioenergetics. Arch Toxicol 2003;77:194-200.

20. Oliveira PJ, Rolo AP, Seiça R, Santos MS, Palmeira CM, Moreno AJ. Chronic cholestasis and cardiac mitochondrial function in Wistar rats: a model for cardiovascular alterations in chronic liver disease? Rev Port Cardiol 2003;22:67-75.

21. Silva VM, Hennig GE, Manautou JE. Cholestasis induced by model organic anions protects from acetaminophen hepatotoxicity in male CD-1 mice. Toxicol Lett 2006;160:204-11.

22. Manautou JE, Hoivik DJ, Tveit A, Hart SG, Khairallah EA, Cohen SD. Clofibrate pretreatment diminishes acetaminophen's selective covalent binding and hepatotoxicity. Toxicol Appl Pharmacol 1994;129:252-63.

23. Maillette de Buy Wenniger L, Beuers U. Bile salts and cholestasis. Dig Liver Dis 2010;42:409-18.

24. Tanaka Y, Aleksunes LM, Cui YJ, Klaassen CD. ANIT-induced intrahepatic cholestasis alters hepatobiliary transporter expression via Nrf2-dependent and independent signaling. Toxicol Sci 2009;108:247-57.

25. Keith CT, Borisy AA, Stockwell BR. Multicomponent therapeutics for networked systems. Nat Rev Drug Discov 2005;4:71-8.

26. Wang X, Zhang A, Wang P, et al. Metabolomics coupled with proteomics advancing drug discovery toward more agile development of targeted combination therapies. Mol Cell Proteomics 2013;12:1226-38.

27. Murat Bilgin H, Atmaca M, Deniz Obay B, Ozekinci S, Taşdemir E, Ketani A. Protective effects of coumarin and coumarin derivatives against carbon tetrachloride-induced acute hepatotoxicity in rats. Exp Toxicol Pathol 2011;63:325-30.

28. Ma T, Huang C, Zong G, et al. Hepatoprotective effects of geniposide in a rat model of nonalcoholic steatohepatitis. J Pharm Pharmacol 2011;63:587-93.

29. Yin F, Liu J, Zheng X, Guo L, Xiao H. Geniposide induces the expression of heme oxygenase-1 via PI3K/Nrf2-signaling to enhance the antioxidant capacity in primary hippocampal neurons. Biol Pharm Bull 2010;33: 1841-6.

30. Guo MZ, Li XS, Xu HR, Mei ZC, Shen W, Ye XF. Rhein inhibits liver fibrosis induced by carbon tetrachloride in rats. Acta Pharmacol Sin 2002;23: 739-44.

31. Sheng X, Wang M, Lu M, Xi B, Sheng H, Zang YQ. Rhein ameliorates fatty liver disease through negative energy balance, hepatic lipogenic regulation, and immunomodulation in diet-induced obese mice. Am J Physiol Endocrinol Metab 2011;300:E886-93.

32. Lin YL, Wu CF, Huang YT. Phenols from the roots of Rheum palmatum attenuate chemotaxis in rat hepatic stellate cells. Planta Med 2008;74: 1246-52.

33. Pan TL, Wang PW, Leu YL, Wu TH, Wu TS. Inhibitory effects of Scutellaria baicalensis extract on hepatic stellate cells through inducing G2/M cell cycle arrest and activating ERK-dependent apoptosis via Bax and caspase pathway. J Ethnopharmacol 2012;139:829-37.

34. Wang MH, Li LZ, Sun JB, Wu FH, Liang JY. A new antioxidant flavone glycoside from Scutellaria baicalensis Georgi. Nat Prod Res 2014;28:1772-6.

35. Noe J, Kullak-Ublick GA, Jochum W, et al. Impaired expression and function of the bile salt export pump due to three novel ABCB11 mutations in intrahepatic cholestasis. J Hepatol 2005;43:536-43.

36. Diao L, Li N, Brayman TG, Hotz KJ, Lai Y. Regulation of MRP2/ABCC2 and BSEP/ABCB11 expression in sandwich cultured human and rat hepatocytes exposed to inflammatory cytokines TNF-\{alpha\}, IL-6, and IL1 \{beta\}. J Biol Chem 2010;285:31185-92.

37. Takikawa H. Hepatobiliary transport of bile acids and organic anions. J Hepatobiliary Pancreat Surg 2002;9:443-7.

38. Dietrich CG, Ottenhoff R, de Waart DR, Oude Elferink RP. Role of MRP2 and GSH in intrahepatic cycling of toxins. Toxicology 2001;167:73-81.

39. Zhu XD, Zhang JB, Zhuang PY, et al. High expression of macrophage colony-stimulating factor in peritumoral liver tissue is associated with poor survival after curative resection of hepatocellular carcinoma. J Clin Oncol 2008;26:2707-16.

40. Zinchuk V, Zinchuk O, Akimaru K, Moriya F, Okada T. Ethanol consumption alters expression and colocalization of bile salt export pump and multidrug resistance protein 2 in the rat. Histochem Cell Biol 2007;127:503-12. 